\title{
EXISTENCE OF SOLUTIONS OF MINIMIZATION PROBLEMS WITH AN INCREASING COST FUNCTION AND POROSITY
}

\author{
ALEXANDER J. ZASLAVSKI
}

Received 18 July 2002

We consider the minimization problem $f(x) \rightarrow \min , x \in K$, where $K$ is a closed subset of an ordered Banach space $X$ and $f$ belongs to a space of increasing lower semicontinuous functions on $K$. In our previous work, we showed that the complement of the set of all functions $f$, for which the corresponding minimization problem has a solution, is of the first category. In the present paper we show that this complement is also a $\sigma$-porous set.

\section{Introduction}

The study of a generic existence of solutions in optimization has recently been a rapidly growing area of research (see $[1,2,3,4,5,6,8,9,10,12,13,14,15]$ and the references mentioned there). Instead of considering the existence of solutions for a single cost function, we study it for a space of all such cost functions equipped with an appropriate complete uniformity and show that a solution exists for most of these functions. Namely, we show that in the space of functions, there exists a subset which is a countable intersection of open everywhere dense sets such that for each cost function in this subset, the corresponding minimization problem has a unique solution. This approach allows us to establish the existence of solutions of minimization problems without restrictive assumptions on the functions and on their domains.

Let $K$ be a nonempty closed subset of a Banach ordered space $(X,\|\cdot\|, \geq)$. A function $f: K \rightarrow \mathbb{R}^{1} \cup\{+\infty\}$ is called increasing if

$$
f(x) \leq f(y) \quad \forall x, y \in K \text { such that } x \leq y .
$$

Increasing functions are considered in many models of mathematical economics. As a rule, both utility and production functions are increasing with respect to natural order relations. 
In this paper, we study the existence of a solution of the minimization problem

$$
f(x) \longrightarrow \min , \quad x \in K,
$$

where $f: K \rightarrow \mathbb{R}^{1} \cup\{+\infty\}$ is an increasing lower semicontinuous function. In $[10,12]$, it was established the generic existence of solutions of problem (1.2) for certain classes of increasing lower semicontinuous functions $f$. Note that the perturbations which are usually used to obtain a generic existence result are not suitable for these classes since they break the monotonicity. In [10], we proposed the new kind of perturbations which allowed us to establish the generic existence of solutions for certain classes of increasing lower semicontinuous functions. In the present paper, we show that the complement of the set of all functions $f$, for which the corresponding minimization problem has a solution, is not only of the first category but also $\sigma$-porous.

Before we continue, we briefly recall the concept of porosity $[2,4]$. As a matter of fact, several different notions of porosity have been used in the literature. In the present paper, we will use porosity with respect to a pair of metrics, a concept which was introduced in [15].

When $(Y, d)$ is a metric space, we denote by $B_{d}(y, r)$ the closed ball of center $y \in Y$ and radius $r>0$. Assume that $Y$ is a nonempty set and $d_{1}, d_{2}: Y \times Y \rightarrow$ $[0, \infty)$ are two metrics which satisfy $d_{1}(x, y) \leq d_{2}(x, y)$ for all $x, y \in Y$. A subset $E \subset Y$ is called porous in $Y$ with respect to the pair $\left(d_{1}, d_{2}\right)$ (or just porous in $Y$ if the pair of metrics is understood) if there exist $\alpha \in(0,1)$ and $r_{0}>0$ such that for each $r \in\left(0, r_{0}\right]$ and each $y \in Y$, there exists $z \in Y$ for which $d_{2}(z, y) \leq r$ and $B_{d_{1}}(z, \alpha r) \cap E=\varnothing$. A subset of the space $Y$ is called $\sigma$-porous in $Y$ with respect to $\left(d_{1}, d_{2}\right)$ (or just $\sigma$-porous in $Y$ if the pair of metrics is understood) if it is a countable union of porous (with respect to $\left(d_{1}, d_{2}\right)$ ) subsets of $Y$. Note that if $d_{1}=d_{2}$, then by [15, Proposition 1.1] our definitions reduce to those in $[2,4]$. We use porosity with respect to a pair of metrics because in applications a space is usually endowed with a pair of metrics and one of them is weaker than the other. Note that porosity of a set with respect to one of these two metrics does not imply its porosity with respect to the other metric. However, it is shown in [15, Proposition 1.2] that if a subset $E \subset Y$ is porous in $Y$ with respect to $\left(d_{1}, d_{2}\right)$, then $E$ is porous in $Y$ with respect to any metric which is weaker than $d_{2}$ and stronger than $d_{1}$.

We obtain our main results as a realization of a general variational principle which is established in Section 3.

\section{Well-posedness of optimization problems with increasing cost functions}

In this paper, we use the following notations and definitions. Let $(X,\|\cdot\|, \geq)$ be a Banach ordered space and $X_{+}=\{x \in X: x \geq 0\}$ the cone of its positive elements. Assume that $X_{+}$is a closed convex cone such that $\|x\| \leq\|y\|$ for each $x, y \in X_{+}$ satisfying $x \leq y$. We assume that the cone $X_{+}$has the following property: 
(A) if $\left\{x_{i}\right\}_{i=1}^{\infty} \subset X, x_{i+1} \leq x_{i}$, for all integers $i \geq 1$ and $\sup \left\{\left\|x_{i}\right\|: i=1,2, \ldots\right\}<$ $\infty$, then the sequence $\left\{x_{i}\right\}_{i=1}^{\infty}$ converges.

The property (A) is well known in the theory of ordered Banach spaces (see, e.g., $[7,10,11])$. Recall that the cone $X_{+}$has the property (A) if the space $X$ is reflexive. The property (A) also holds for the cone of nonnegative functions (with respect to usual order relation) in the space $L_{1}$ of all integrable on a measure space functions.

Assume that $K$ is a closed subset of $X$. For each function $f: Y \rightarrow[-\infty,+\infty]$, where $Y$ is a nonempty set, we define

$$
\operatorname{dom}(f)=\{y \in Y:|f(y)|<\infty\}, \quad \inf (f)=\inf \{f(y): y \in Y\} .
$$

We use the convention that $\infty-\infty=0, \infty / \infty=1$, and $\ln (\infty)=\infty$.

Assume that $\mathscr{A}$ is a nonempty set and $d_{w}, d_{s}: \mathscr{A} \times \mathscr{A} \rightarrow[0, \infty)$ are two metrics which satisfy $d_{w}(a, b) \leq d_{s}(a, b)$ for all $a, b \in \mathscr{A}$. We assume that the metric space $\left(\mathscr{A}, d_{s}\right)$ is complete. The topology induced in $\mathscr{A}$ by the metric $d_{s}$ is called the strong topology and the topology induced in $\mathscr{A}$ by the metric $d_{w}$ is called the weak topology.

We assume that with every $a \in \mathscr{A}$ a lower semicontinuous function $f_{a}: K \rightarrow$ $[-\infty,+\infty]$ is associated and $f_{a}$ is not identically $\infty$ for all $a \in \mathscr{A}$.

Let $a \in \mathscr{A}$. We say that the minimization problem for $f_{a}$ on $K$ is strongly well posed with respect to $\left(\mathscr{A}, d_{w}\right)$ if the following assertions hold:

(1) the infimum $\inf \left(f_{a}\right)$ is finite and attained at a point $x^{(a)} \in K$ such that for each $x \in K$ satisfying $f_{a}(x)=\inf \left(f_{a}\right)$, the inequality $x \leq x^{(a)}$ holds;

(2) for any $\epsilon>0$, there exist $\delta>0$ and a neighborhood $U$ of $a$ in $\mathscr{A}$ with the weak topology such that for each $b \in U, \inf \left(f_{b}\right)$ is finite; and if $x \in K$ satisfies $f_{b}(x) \leq \inf \left(f_{b}\right)+\delta$, then $\left|f_{a}\left(x^{(a)}\right)-f_{b}(x)\right|<\epsilon$ and there is $u \in X$ such that $\|u\|<\epsilon$ and $x \leq x^{(a)}+u$.

Note that if $X_{+}=\{0\}$, then our definition reduces to those in $[6,13]$.

For each integer $n \geq 1$, denote by $\mathscr{A}_{n}$ the set of all $a \in \mathscr{A}$ which have the following property:

(P1) there exist $x \in K$ and positive numbers $r, \eta$, and $c$ such that

$$
-\infty<f_{a}(x)<\inf \left(f_{a}\right)+\frac{1}{n}
$$

and for each $b \in \mathscr{A}$ satisfying $d_{w}(a, b)<r, \inf \left(f_{b}\right)$ is finite; and if $z \in$ $K$ satisfies $f_{b}(z) \leq \inf \left(f_{b}\right)+\eta$, then $\|z\| \leq c,\left|f_{b}(z)-f_{a}(x)\right| \leq 1 / n$, and there is $u \in X$ such that $\|u\| \leq 1 / n$ and $z \leq x+u$.

Proposition 2.1. Assume that $a \in \cap_{n=1}^{\infty} \mathscr{A}_{n}$. Then the minimization problem for $f_{a}$ on $K$ is strongly well posed with respect to $\left(\mathscr{A}, d_{w}\right)$. 


\section{Solutions of minimization problems}

Proof. By (P1) for each integer $n \geq 1$, there exist $x_{n} \in K, r_{n}>0, \eta_{n}>0$, and $c_{n}>0$ such that

$$
-\infty<f_{a}\left(x_{n}\right)<\inf \left(f_{a}\right)+2^{-n}
$$

and the following property holds:

(P2) for each $b \in \mathscr{A}$ satisfying $d_{w}(a, b)<r_{n}, \inf \left(f_{b}\right)$ is finite; and if $z \in K$ satisfies $f_{b}(z) \leq \inf \left(f_{b}\right)+\eta_{n}$, then there exists $u \in X$ such that

$$
\|u\| \leq 2^{-n}, \quad z \leq x_{n}+u, \quad\|z\| \leq c_{n}, \quad\left|f_{b}(z)-f_{a}\left(x_{n}\right)\right| \leq 2^{-n} .
$$

We may assume without loss of generality that for all integers $n \geq 1$,

$$
\eta_{n}, r_{n}<4^{-n-1}, \quad \eta_{n}<\eta_{1}
$$

There exists a strictly increasing sequence of natural numbers $\left\{k_{n}\right\}_{n=1}^{\infty}$ such that

$$
4 \cdot 2^{-k_{n+1}}<\eta\left(k_{n}\right) \quad \text { for all integers } n \geq 1 \text {. }
$$

Let $n \geq 1$ be an integer. Inequality (2.3) implies that

$$
-\infty<f_{a}\left(x_{k_{n+1}}\right)<\inf \left(f_{a}\right)+2^{-k_{n+1}}<\inf \left(f_{a}\right)+\eta\left(k_{n}\right) .
$$

By (2.7), (2.5), and the definition of $c_{1}$,

$$
\left\|x_{k_{n+1}}\right\| \leq c_{1}
$$

It follows from (2.7), (P2) (see (2.4)), and the definitions of $x_{k_{n}}$ and $\eta_{k_{n}}$ that there exists $u_{n} \in X$ such that

$$
\begin{array}{r}
\left\|u_{n}\right\| \leq 2^{-k_{n}}, \quad x_{k_{n+1}} \leq x_{k_{n}}+u_{n}, \\
\left|f_{a}\left(x_{k_{n+1}}\right)-f_{a}\left(x_{k_{n}}\right)\right| \leq 2^{-k_{n}} .
\end{array}
$$

Set

$$
y_{n}=x_{k_{n}}+\sum_{i=n}^{\infty} u_{i}
$$

Clearly, the sequence $\left\{y_{n}\right\}_{n=1}^{\infty}$ is well defined. By (2.11) and (2.9), for each integer $n \geq 1$,

$$
y_{n+1}-y_{n}=x_{k_{n+1}}+\sum_{i=n+1}^{\infty} u_{i}-\left(x_{k_{n}}+\sum_{i=n}^{\infty} u_{i}\right)=x_{k_{n+1}}-x_{k_{n}}-u_{n} \leq 0 .
$$


Equation (2.11) and inequalities (2.9) and (2.8) imply that

$$
\sup \left\{\left\|y_{n}\right\|: n=1,2, \ldots\right\}<\infty
$$

It follows from (2.13), (2.12), and the property (A) that there is $x^{(a)}=\lim _{n \rightarrow \infty} y_{n}$. Combined with (2.11) and (2.9), this equality implies that

$$
x^{(a)}=\lim _{n \rightarrow \infty} x_{k_{n}}
$$

By (2.14), (2.3), and the lower semicontinuity of $f_{a}$,

$$
f_{a}\left(x^{(a)}\right)=\inf \left(f_{a}\right)
$$

Assume now that $x \in K$ and $f_{a}(x)=\inf \left(f_{a}\right)$. By the definition of $x_{k_{n}}, n=$ $1,2, \ldots$ (see the property (P2)), for each integer $n \geq 1$, there is $v_{n} \in X$ such that

$$
\left\|v_{n}\right\| \leq 2^{-k_{n}}, \quad x \leq x_{k_{n}}+v_{n} .
$$

These inequalities and (2.14) imply that $x \leq x^{(a)}$.

By (2.15) and the property (P2), for all integers $n \geq 1$,

$$
\left|f_{a}\left(x^{(a)}\right)-f_{a}\left(x_{n}\right)\right| \leq 2^{-n}
$$

Let $\epsilon>0$. Choose a natural number $m$ for which

$$
\left\|x^{(a)}-x_{k_{m}}\right\|<4^{-1} \epsilon, \quad 2^{-k_{m}}<4^{-1} \epsilon .
$$

Assume that $b \in B_{w}\left(a, r_{k_{m}} / 2\right), x \in K$, and

$$
f_{b}(x) \leq \inf \left(f_{b}\right)+\eta_{k_{m}}
$$

By (2.19) and the definitions of $\eta_{k_{m}}, r_{k_{m}}$, and $x_{k_{m}}$ (see the property (P2)),

$$
\left|f_{b}(x)-f_{a}\left(x_{k_{m}}\right)\right| \leq 2^{-k_{m}}
$$

and there is $v \in X$ such that

$$
\|v\|<2^{-k_{m}}, \quad x \leq x_{k_{m}}+v
$$


It follows from (2.21) and (2.18) that

$$
\begin{gathered}
x \leq x_{k_{m}}+v=x^{(a)}+\left(x_{k_{m}}-x^{(a)}+v\right), \\
\left\|x_{k_{m}}-x^{(a)}+v\right\| \leq\left\|x^{(a)}-x_{k_{m}}\right\|+\|v\|<\frac{\epsilon}{2} .
\end{gathered}
$$

Inequalities (2.20), (2.17), and (2.18) imply that

$$
\begin{aligned}
\left|f_{a}\left(x^{(a)}\right)-f_{b}(x)\right| & \leq\left|f_{a}\left(x^{(a)}\right)-f_{a}\left(x_{k_{m}}\right)\right|+\left|f_{a}\left(x_{k_{m}}\right)-f_{b}(x)\right| \\
& \leq 2^{-k_{m}}+2^{-k_{m}}<\frac{\epsilon}{2} .
\end{aligned}
$$

This completes the proof of Proposition 2.1.

An element $x \in K$ is called minimal if for each $y \in K$ satisfying $y \leq x$, the equality $x=y$ is true. Denote by $K_{\min }$ the set of all minimal elements of $K$.

For each integer $n \geq 1$, denote by $\tilde{A}_{n}$ the set of all $a \in \mathscr{A}$ which has the property (P1) with $x \in K_{\min }$.

Analogously to the proof of Proposition 2.1, we can prove the following result.

Proposition 2.2. Assume that the set $K_{\min }$ is a closed subset of the Banach space $X$ and $a \in \cap_{n=1}^{\infty} \tilde{A}_{n}$. Then the minimization problem for $f_{a}$ on $K$ is strongly well posed with respect to $\left(\mathscr{A}, d_{w}\right)$ and $\inf \left(f_{a}\right)$ is attained at a unique point.

In the proof of Proposition 2.2, we choose $x_{n} \in K_{\min }, n=1,2, \ldots$ This implies that $\inf \left(f_{a}\right)$ is attained at the unique point $x^{(a)} \in K_{\min }$ (see (2.13)).

Remark 2.3. Note that assertion (1) in the definition of a strongly well-posed minimization problem for $f_{a}$ can be represented in the following way: $\inf \left(f_{a}\right)$ is finite and the set

$$
\underset{x \in K}{\operatorname{argmin}} f_{a}=\left\{x \in K: f_{a}(x)=\inf \left(f_{a}\right)\right\}
$$

has the largest element.

We construct an example of an increasing function $h$ for which the set $\operatorname{argmin}(h)$ is not a singleton and has the largest element. Define a continuous increasing function $\psi:[0, \infty) \rightarrow \mathbb{R}^{1}$ by

$$
\psi(t)=0, \quad t \in\left[0, \frac{1}{2}\right], \quad \psi(t)=2 t-1, \quad t \in\left(\frac{1}{2}, \infty\right) .
$$

Let $n$ be a natural number and consider the Euclidean space $\mathbb{R}^{n}$. Let $K=\{x=$ $\left.\left(x_{1}, \ldots, x_{n}\right) \in \mathbb{R}^{n}: x_{i} \geq 0, i=1, \ldots, n\right\}$. Define a function $h: K \rightarrow \mathbb{R}^{1}$ by

$$
h(x)=\psi\left(\max \left\{x_{i}: i=1, \ldots, n\right\}\right), \quad x \in K
$$


It is easy to see that $h$ is a continuous increasing function:

$$
\inf \{h(x): x \in K\}=0
$$

and the set

$$
\{x \in K: h(x)=0\}=\left\{x=\left(x_{1}, \ldots, x_{n}\right) \in \mathbb{R}^{n}: x_{i} \in\left[0, \frac{1}{2}\right], i=1, \ldots, n\right\}
$$

is not a singleton and has the largest element $(1 / 2, \ldots, 1 / 2)$.

Remark 2.4. The following example shows that in some cases the sets $\mathscr{A}_{n}$ can be empty. Let $\mathscr{A}=K=\mathbb{R}^{1}$. For each $a \in \mathbb{R}^{1}$, consider the function $f_{a}: K \rightarrow \mathbb{R}^{1}$, where $f_{a}=0$ for any $x \leq a$ and $f_{a}(x)>0$ for any $x>a$. It is easy to see that the set $\mathscr{A}_{n}$ is empty for any natural number $n$.

\section{Variational principles}

We use the notations and definitions introduced in Section 2. The following are the basic hypotheses about the functions:

(H1) for each $a \in \mathscr{A}, \inf \left(f_{a}\right)$ is finite;

(H2) for each $\epsilon>0$ and each integer $m \geq 1$, there exist numbers $\delta>0$ and $r_{0}>0$ such that the following property holds:

(P3) for each $a \in \mathscr{A}$ satisfying $\inf \left(f_{a}\right) \leq m$ and each $r \in\left(0, r_{0}\right]$, there exist $\bar{a} \in \mathscr{A}, \bar{x} \in K$, and $\bar{d}>0$ such that

$$
d_{s}(a, \bar{a}) \leq r, \quad \inf \left(f_{\bar{a}}\right) \leq m+1, \quad f_{\bar{a}}(\bar{x}) \leq \inf \left(f_{\bar{a}}\right)+\epsilon,
$$

and if $x \in K$ satisfies

$$
f_{\bar{a}}(x) \leq \inf \left(f_{\bar{a}}\right)+\delta r,
$$

then $\|x\| \leq \bar{d}$ and there exists $u \in X$ for which $\|u\| \leq \epsilon$ and $x \leq \bar{x}+u$;

(H3) for each integer $m \geq 1$, there exist $\alpha \in(0,1)$ and $r_{0}>0$ such that for each $r \in\left(0, r_{0}\right]$, each $a_{1}, a_{2} \in \mathscr{A}$ satisfying $d_{w}\left(a_{1}, a_{2}\right) \leq \alpha r$, and each $x \in$ $K$ satisfying $\min \left\{f_{a_{1}}(x), f_{a_{2}}(x)\right\} \leq m$, the inequality $\left|f_{a_{1}}(x)-f_{a_{2}}(x)\right| \leq r$ is valid.

Theorem 3.1. Assume that (H1), (H2), and (H3) hold. Then there exists a set $\mathscr{F} \subset \mathscr{A}$ such that the complement $\mathscr{A} \backslash \mathscr{F}$ is $\sigma$-porous in $\mathscr{A}$ with respect to $\left(d_{w}, d_{s}\right)$ and for each $a \in \mathscr{F}$, the minimization problem for $f_{a}$ on $K$ is strongly well posed with respect to $\left(\mathscr{A}, d_{w}\right)$.

Proof. Recall that for each integer $n \geq 1, \mathscr{A}_{n}$ is the set of all $a \in \mathscr{A}$ which has the property (P1). By Proposition 2.1, in order to prove the theorem, it is sufficient to show that the set $\mathscr{A} \backslash \mathscr{A}_{n}$ is $\sigma$-porous in $\mathscr{A}$ with respect to $\left(d_{w}, d_{s}\right)$ for any integer $n \geq 1$. Then the theorem is true with $\mathscr{F}=\cap_{n=1}^{\infty} \mathscr{A}_{n}$. 
Let $n \geq 1$ be an integer. We will show that the set $\mathscr{A} \backslash \mathscr{A}_{n}$ is $\sigma$-porous in $\mathscr{A}$ with respect to $\left(d_{w}, d_{s}\right)$. To meet this goal, it is sufficient to show that for each integer $m \geq 1$, the set

$$
\Omega_{n m}:=\left\{a \in \mathscr{A} \backslash \mathscr{A}_{n}: \inf \left(f_{a}\right) \leq m\right\}
$$

is porous in $\mathscr{A}$ with respect to $\left(d_{w}, d_{s}\right)$.

Let $m \geq 1$ be an integer. By (H3), there exist

$$
\alpha_{1} \in(0,1), \quad r_{1} \in\left(0, \frac{1}{2}\right)
$$

such that for each $r \in\left(0, r_{1}\right]$, each $a_{1}, a_{2} \in \mathscr{A}$ satisfying $d_{w}\left(a_{1}, a_{2}\right) \leq \alpha_{1} r$, and each $x \in K$ satisfying

$$
\min \left\{f_{a_{1}}(x), f_{a_{2}}(x)\right\} \leq m+4
$$

the inequality $\left|f_{a_{1}}(x)-f_{a_{2}}(x)\right| \leq r$ holds.

By (H2), there exist $\alpha_{2}, r_{2} \in(0,1)$ such that the following property holds:

(P4) for each $a \in \mathscr{A}$ satisfying $\inf \left(f_{a}\right) \leq m+2$ and each $r \in\left(0, r_{2}\right]$, there exist $\bar{a} \in \mathscr{A}, \bar{x} \in K$, and $\bar{d}>0$ such that

$$
d_{s}(a, \bar{a}) \leq r, \quad \inf \left(f_{\bar{a}}\right) \leq m+3, \quad f_{\bar{a}}(\bar{x}) \leq \inf \left(f_{\bar{a}}\right)+(2 n)^{-1},
$$

and if $x \in K$ satisfies $f_{\bar{a}}(x) \leq \inf \left(f_{\bar{a}}\right)+4 r \alpha_{2}$, then $\|x\| \leq \bar{d}$ and there exists $u \in X$ for which $\|u\| \leq(2 n)^{-1}$ and $x \leq \bar{x}+u$.

Choose

$$
\bar{\alpha} \in\left(0, \frac{\alpha_{1} \alpha_{2}}{16}\right), \quad \bar{r} \in\left(0, \frac{r_{1} r_{2} \bar{\alpha}}{n}\right) .
$$

Let $a \in \mathscr{A}$ and $r \in(0, \bar{r}]$. There are two cases

$$
\begin{aligned}
& B_{d_{s}}\left(a, \frac{r}{4}\right) \cap\left\{\xi \in \mathscr{A}: \inf \left(f_{\xi}\right) \leq m+2\right\}=\varnothing, \\
& B_{d_{s}}\left(a, \frac{r}{4}\right) \cap\left\{\xi \in \mathscr{A}: \inf \left(f_{\xi}\right) \leq m+2\right\} \neq \varnothing .
\end{aligned}
$$

Assume that (3.8) holds. We will show that for each $\xi \in B_{d_{w}}(a, \bar{r})$, the inequality $\inf \left(f_{\xi}\right)>m$ is valid. Assume the contrary. Then there exists $\xi \in \mathscr{A}$ such that

$$
d_{w}(\xi, a) \leq \bar{r}, \quad \inf \left(f_{\xi}\right) \leq m .
$$

There exists $y \in K$ such that

$$
f_{\xi}(y) \leq m+\frac{1}{2}
$$


It follows from the definitions of $\alpha_{1}, r_{1}$ (see (3.4), (3.5)), (3.11), (3.10), and (3.7) that

$$
\left|f_{a}(y)-f_{\xi}(y)\right| \leq \alpha_{1}^{-1} \bar{r} \leq \frac{1}{4}
$$

This inequality and (3.11) imply that

$$
\inf \left(f_{a}\right) \leq f_{a}(y) \leq f_{\xi}(y)+\frac{1}{4} \leq m+1,
$$

a contradiction (see (3.8)). Therefore

$$
B_{d_{w}}(a, \bar{r}) \subset\left\{\xi \in \mathscr{A}: \inf \left(f_{\xi}\right)>m\right\}
$$

and by (3.3),

$$
B_{d_{w}}(a, \bar{r}) \cap \Omega_{n m}=\varnothing .
$$

Thus, we have shown that (3.8) implies (3.15).

Assume that (3.9) holds. Then there exists $a_{1} \in \mathscr{A}$ such that

$$
d_{s}\left(a, a_{1}\right) \leq \frac{r}{4}, \quad \inf \left(f_{a_{1}}\right) \leq m+2 .
$$

By the definitions of $\alpha_{2}, r_{2}$, the property (P4), (3.16), and (3.7), there exist $\bar{a} \in \mathscr{A}$, $\bar{x} \in K, \bar{d}>0$ such that

$$
d_{s}\left(a_{1}, \bar{a}\right) \leq \frac{r}{4}, \quad \inf \left(f_{\bar{a}}\right) \leq m+3, \quad f_{\bar{a}}(\bar{x}) \leq \inf \left(f_{\bar{a}}\right)+(2 n)^{-1}
$$

and that the following property holds:

(P5) if $x \in K$ satisfies $f_{\bar{a}}(x) \leq \inf \left(f_{\bar{a}}\right)+r \alpha_{2}$, then $\|x\| \leq \bar{d}$ and there exists $u \in$ $X$ for which $\|u\| \leq(2 n)^{-1}$ and $x \leq \bar{x}+u$.

Inequalities (3.17) and (3.16) imply that

$$
d_{s}(a, \bar{a}) \leq \frac{r}{2}
$$

Assume that

$$
\xi \in B_{d_{w}}(\bar{a}, \bar{\alpha} r) .
$$

By (3.17),

$$
\inf \left(f_{\bar{a}}\right)=\inf \left\{f_{\bar{a}}(z): z \in K, f_{\bar{a}}(z) \leq m+\frac{7}{2}\right\}
$$

Let $x \in K$ satisfy

$$
f_{\bar{a}}(x) \leq m+\frac{7}{2}
$$


It follows from (3.19), (3.21), (3.7), and the definitions of $\alpha_{1}, r_{1}$ (see (3.4), (3.5)) that

$$
\left|f_{\bar{a}}(x)-f_{\xi}(x)\right| \leq \bar{\alpha} r \alpha_{1}^{-1} \leq \frac{\alpha_{2} r}{16} .
$$

Since these inequalities hold for any $x \in K$ satisfying (3.21), the relation (3.20) implies that

$$
\begin{aligned}
\inf \left(f_{\xi}\right) & \leq \inf \left\{f_{\xi}(x): x \in K, f_{\bar{a}}(x) \leq m+\frac{7}{2}\right\} \\
& \leq \inf \left\{f_{\bar{a}}(x)+\frac{\alpha_{2} r}{16}: x \in K, f_{\bar{a}}(x) \leq m+\frac{7}{2}\right\} \\
& =\frac{\alpha_{2} r}{16}+\inf \left(f_{\bar{a}}\right) .
\end{aligned}
$$

Moreover, since (3.21) holds with $x=\bar{x}$ (see (3.17)), we obtain that $\mid f_{\bar{a}}(\bar{x})-$ $f_{\xi}(\bar{x}) \mid \leq \alpha_{2} r / 16$. Thus

$$
\inf \left(f_{\bar{\xi}}\right) \leq \inf \left(f_{\bar{a}}\right)+\frac{\alpha_{2} r}{16}, \quad\left|f_{\bar{a}}(\bar{x})-f_{\xi}(\bar{x})\right| \leq \frac{\alpha_{2} r}{16} .
$$

Let $x \in K$ satisfy

$$
f_{\xi}(x) \leq \inf \left(f_{\xi}\right)+\frac{1}{4}
$$

Inequalities (3.25), (3.24), (3.17) and (3.7) imply that $f_{\xi}(x) \leq m+7 / 2$. It follows from this inequality, (3.19), (3.7), and the definitions of $\alpha_{1}, r_{1}$ (see (3.4), (3.5)) that

$$
\left|f_{\bar{a}}(x)-f_{\xi}(x)\right| \leq \bar{\alpha} r \alpha_{1}^{-1} \leq \frac{\alpha_{2} r}{16} .
$$

Thus, the following property holds:

(P6) if $x \in K$ satisfies (3.25), then $\left|f_{\bar{a}}(x)-f_{\xi}(x)\right| \leq \alpha_{2} r / 16$.

The property (P6) implies that

$$
\begin{aligned}
\inf \left(f_{\bar{a}}\right) & \leq \inf \left\{f_{\bar{a}}(x): x \in K, f_{\xi}(x) \leq \inf \left(f_{\xi}\right)+\frac{1}{4}\right\} \\
& \leq \inf \left\{f_{\xi}(x)+\frac{\alpha_{2} r}{16}: x \in K, f_{\xi}(x) \leq \inf \left(f_{\xi}\right)+\frac{1}{4}\right\} \\
& =\frac{\alpha_{2} r}{16}+\inf \left(f_{\xi}\right) .
\end{aligned}
$$

Therefore

$$
\inf \left(f_{\bar{a}}\right) \leq \inf \left(f_{\xi}\right)+\frac{\alpha_{2} r}{16}
$$


Combined with (3.24) and (3.17), this inequality implies that

$$
\left|\inf \left(f_{\bar{a}}\right)-\inf \left(f_{\xi}\right)\right| \leq \frac{\alpha_{2} r}{16}, \quad f_{\xi}(\bar{x}) \leq \inf \left(f_{\xi}\right)+\frac{\alpha_{2} r}{8}+(2 n)^{-1} .
$$

Assume that $x \in K$ and

$$
f_{s}(x) \leq \inf \left(f_{\xi}\right)+\frac{\alpha_{2} r}{16} .
$$

By (P6),

$$
\left|f_{\bar{a}}(x)-f_{\xi}(x)\right| \leq \frac{\alpha_{2} r}{16} .
$$

Inequalities (3.30), (3.29), (3.17) and (3.7) imply that

$$
\begin{aligned}
\left|f_{\xi}(x)-f_{\bar{a}}(\bar{x})\right| \leq & \left|f_{\xi}(x)-\inf \left(f_{\xi}\right)\right|+\left|\inf \left(f_{\xi}\right)-\inf \left(f_{\bar{a}}\right)\right| \\
& +\left|\inf \left(f_{\bar{a}}\right)-f_{\bar{a}}(\bar{x})\right| \\
\leq & \frac{\alpha_{2} r}{16}+\frac{\alpha_{2} r}{16}+(2 n)^{-1}<n^{-1} \\
& \left|f_{\xi}(x)-f_{\bar{a}}(\bar{x})\right|<n^{-1}
\end{aligned}
$$

It follows from (3.31), (3.30), and (3.29) that

$$
\begin{aligned}
f_{\bar{a}}(x) \leq f_{\xi}(x)+\frac{r \alpha_{2}}{16} & \leq \inf \left(f_{\xi}\right)+\frac{\alpha_{2} r}{8} \leq \inf \left(f_{\bar{a}}\right)+\frac{3 \alpha_{2} r}{16}, \\
f_{\bar{a}}(x) & \leq \inf \left(f_{\bar{a}}\right)+\frac{3 \alpha_{2} r}{16} .
\end{aligned}
$$

It follows from (3.35) and the property (P5) that $\|x\| \leq \bar{d}$ and there is $u \in X$ such that $\|u\| \leq(2 n)^{-1}$ and $x \leq u+\bar{x}$. Therefore, if $x \in K$ satisfies (3.30), then (3.33) is valid, $\|x\| \leq \bar{d}$, and there is $u \in X$ for which $\|u\| \leq(2 n)^{-1}$ and $x \leq u+\bar{x}$.

Thus, we have shown that for each $\xi \in B_{d_{w}}(\bar{a}, \bar{\alpha} r)$, the inequalities (3.29) are true and if $x \in K$ satisfies (3.30), then (3.33) is valid, $\|x\| \leq \bar{d}$, and there is $u \in X$ for which $\|u\| \leq(2 n)^{-1}$ and $x \leq u+\bar{x}$.

By the definition of $\mathscr{A}_{n}$ and (3.3),

$$
B_{d_{w}}\left(\bar{a}, \frac{\bar{\alpha} r}{2}\right) \subset \mathscr{A}_{n} \subset \mathscr{A} \backslash \Omega_{n m}
$$

Since (3.8) implies (3.15), we obtain that, in both cases, $B_{d_{w}}(\bar{a}, \bar{\alpha} r / 2) \cap \Omega_{n m}=\varnothing$ with $\bar{a} \in \mathscr{A}$ satisfying (3.18). (Note that if (3.8) is valid, then $\bar{a}=a$.) Hence, the set $\Omega_{n m}$ is porous in $\mathscr{A}$ with respect to $\left(d_{w}, d_{s}\right)$. This implies that $\mathscr{A} \backslash \mathscr{A}_{n}$ is $\sigma$-porous in $\mathscr{A}$ with respect to $\left(d_{w}, d_{s}\right)$ for all integers $n \geq 1$. Therefore, $\mathscr{A} \backslash$ $\left(\cap_{n=1}^{\infty} \mathscr{A}_{n}\right)$ is $\sigma$-porous in $\mathscr{A}$ with respect to $\left(d_{w}, d_{s}\right)$. This completes the proof of Theorem 3.1.

We also use the following hypotheses about the functions: 
(H4) for each $\epsilon>0$ and each integer $m \geq 1$, there exist numbers $\delta>0$ and $r_{0}>0$ such that the following property holds:

(P7) for each $a \in \mathscr{A}$ satisfying $\inf \left(f_{a}\right) \leq m$ and each $r \in\left(0, r_{0}\right]$, there exist $\bar{a} \in \mathscr{A}, \bar{x} \in K_{\min }$, and $\bar{d}>0$ such that (3.1) is true; and if $x \in K$ satisfies (3.2), then $\|x\| \leq \bar{d}$ and there exists $u \in X$ for which $\|u\| \leq \epsilon, x \leq$ $\bar{x}+u$.

Theorem 3.2. Assume that (H1), (H3), and (H4) hold and $K_{\min }$ is a closed subset of the Banach space X. Then there exists a set $\mathscr{F} \subset A$ such that the complement $\mathscr{A} \backslash \mathscr{F}$ is $\sigma$-porous in $\mathscr{A}$ with respect to $\left(d_{w}, d_{s}\right)$ and that for each $a \in \mathscr{F}$ the following assertions hold:

(1) the minimization problem for $f_{a}$ on $K$ is strongly well posed with respect to $\left(\mathcal{A}, d_{w}\right)$,

(2) the infimum $\inf \left(f_{a}\right)$ is attained at a unique point.

We can prove Theorem 3.2 analogously to the proof of Theorem 3.1. Recall that for each integer $n \geq 1, \tilde{A}_{n}$ is the set of all $a \in \mathscr{A}$ which have the property (P1) with $x \in K_{\min }$. Set $\mathscr{F}_{F}=\cap_{n=1}^{\infty} \tilde{A}_{n}$. By Proposition 2.2 for each $a \in \mathscr{F}$, assertions (1) and (2) hold. Therefore, in order to prove Theorem 3.2, it is sufficient to show that for each integer $n \geq 1$, the set $\mathscr{A} \backslash \tilde{A}_{n}$ is $\sigma$-porous in $\mathscr{A}$ with respect to $\left(d_{w}, d_{s}\right)$. We can show this fact analogously to the proof of Theorem 3.1.

\section{Spaces of increasing functions}

In the sequel, we use the functional $\lambda: X \rightarrow \mathbb{R}^{1}$ defined by

$$
\lambda(x)=\inf \{\|y\|: y \geq x\}, \quad x \in X
$$

The function $\lambda$ has the following properties (see [10, Proposition 6.1]):

(i) the function $\lambda$ is sublinear. Namely,

$$
\begin{array}{r}
\lambda(\alpha x)=\alpha \lambda(x) \quad \forall \alpha \geq 0 \text { and all } x \in X, \\
\lambda\left(x_{1}+x_{2}\right) \leq \lambda\left(x_{1}\right)+\lambda\left(x_{2}\right) \quad \forall x_{1}, x_{2} \in X,
\end{array}
$$

(ii) $\lambda(x)=0$ if $x \leq 0$,

(iii) if $x_{1}, x_{2} \in X$ and $x_{1} \leq x_{2}$, then $\lambda\left(x_{1}\right) \leq \lambda\left(x_{2}\right)$,

(iv) $0 \leq \lambda(x) \leq\|x\|$ for all $x \in X$.

Clearly, $|\lambda(x)-\lambda(y)| \leq\|x-y\|$ for each $x, y \in X$.

Denote by $M$ the set of all increasing lower semicontinuos bounded-frombelow functions $f: K \rightarrow \mathbb{R}^{1} \cup\{+\infty\}$ which are not identically $+\infty$. For each $f, g \in M$, set

$$
\begin{aligned}
& \tilde{d}_{s}(f, g)=\sup \{|f(x)-g(x)|: x \in X\}, \\
& d_{s}(f, g)=\tilde{d}_{s}(f, g)\left(1+\tilde{d}_{s}(f, g)\right)^{-1} .
\end{aligned}
$$


It is not difficult to see that the metric space $\left(\mathcal{M}, d_{s}\right)$ is complete. Denote by $\mathcal{M}_{v}$ the set of all finite-valued functions $f \in M$ and by $M_{c}$ the set of all finite-valued continuous functions $f \in M$. Clearly, $\mathcal{M}_{v}$ and $M_{c}$ are closed subsets of the metric space $\left(M, d_{s}\right)$.

We say that the set $K$ has property (C) if $K_{\min }$ is a closed subset of $K$ and for each $x \in K$, there is $y \in K_{\min }$ such that $y \leq x$.

Denote by $M_{g}$ the set of all $f \in \mathcal{M}$ such that $f(x) \rightarrow \infty$ as $\|x\| \rightarrow \infty$. Clearly, $M_{g}$ is a closed subset of the metric space $\left(\mathcal{M}, d_{s}\right)$. Set $M_{g c}=M_{g} \cap M_{c}$ and $M_{g v}=$ $M_{g} \cap M_{v}$.

It is easy to see that

$$
M_{c} \subset M_{v} \subset \mathcal{M}, \quad M_{g c} \subset M_{g v} \subset M_{g} \subset \mathcal{M} .
$$

Remark 4.1. Let $K=X_{+}$and define

$$
\begin{array}{cl}
f_{1}(x)=\|x\|, & x \in K, \quad f_{2}(x)=\|x\|, \quad x \in K \backslash\{0\}, \quad f_{2}(0)=-1, \\
f_{3}(x)=\|x\| & \text { if } x \in K,\|x\| \leq 1, \quad f_{3}(x)=+\infty \quad \text { if } x \in K, \quad\|x\|>1 .
\end{array}
$$

Clearly,

$$
f_{1} \in M_{g c}, \quad f_{2} \in M_{g v} \backslash M_{g c}, \quad f_{3} \in M_{g} \backslash M_{g v} .
$$

Theorem 4.2. Assume that $\mathcal{A}$ is either $\mathcal{M}_{g}, \mathcal{M}_{g v}$, or $\mathcal{M}_{g c}$ and that $f_{a}=a$ for all $a \in \mathscr{A}$. Then there exists a set $\mathscr{F} \subset \mathcal{A}$ such that the complement $\mathscr{A} \backslash \mathscr{F}$ is $\sigma$-porous in $\mathcal{A}$ with respect to $\left(d_{s}, d_{s}\right)$ and that for each $f \in \mathscr{F}$ the minimization problem for $f$ on $K$ is strongly well posed with respect to $\left(\mathscr{A}, d_{s}\right)$. If $K$ has the property $(C)$, then for each $f \in \mathscr{F}, \inf (f)$ is attained at a unique point.

Proof. By Theorems 3.1 and 3.2, we need to show that (H1), (H2), and (H3) hold and that the property (C) implies (H4). Clearly, (H1) holds. For each $f, g \in \mathcal{M}$, we have that

$$
\tilde{d}_{s}(f, g)=d_{s}(f, g)\left(1-d_{s}(f, g)\right)^{-1}
$$

and that if $d_{s}(f, g) \leq 1 / 2$, then $\tilde{d}_{s}(f, g) \leq 2 d_{s}(f, g)$. Combined with (4.7), this property implies $(\mathrm{H} 3)$.

We will show that (H2) holds and that the property (C) implies (H4).

Let $f \in \mathscr{A}, \epsilon \in(0,1)$, and $r \in(0,1]$. Choose $\bar{x} \in K$ such that

$$
f(\bar{x}) \leq \inf (f)+\frac{\epsilon r}{8} .
$$

If $K$ has the property (C), then we assume that $\bar{x}$ is a minimal element of $K$. Define

$$
\bar{f}(x)=f(x)+2^{-1} r \min \{1, \lambda(x-\bar{x})\} \quad \forall x \in K .
$$


Evidently, $\bar{f} \in \mathscr{A}, d_{s}(f, \bar{f}) \leq \tilde{d}_{s}(f, \bar{f}) \leq r / 2$, and

$$
\inf (\bar{f}) \leq \bar{f}(\bar{x})=f(\bar{x}) \leq \inf (f)+\frac{\epsilon r}{8} .
$$

Let $x \in K$ and $\bar{f}(x) \leq \inf (\bar{f})+\epsilon r / 8$. Then by (4.9) and (4.8),

$$
\begin{aligned}
f(x)+2^{-1} r \min \{1, \lambda(x-\bar{x})\} & =\bar{f}(x) \leq \inf (\bar{f})+\frac{\epsilon r}{8} \leq \bar{f}(\bar{x})+\frac{\epsilon r}{8} \\
& =f(\bar{x})+\frac{\epsilon r}{8} \leq f(x)+\frac{\epsilon r}{4}, \\
\min \{1, \lambda(x-\bar{x})\} & \leq \frac{\epsilon}{2}, \quad \lambda(x-\bar{x}) \leq \frac{\epsilon}{2} .
\end{aligned}
$$

By (4.1), there exists $u \in X$ such that $x \leq \bar{x}+u$ and $\|u\|<\epsilon$. Since $\bar{f}(y) \rightarrow \infty$ as $\|y\| \rightarrow \infty$, we obtain that $\|x\| \leq \bar{d}$, where $\bar{d}>0$ is a constant which depends only on $\bar{f}$. Thus, (H2) is true and if $K$ has the property (C), then (H4) holds. Theorem 4.2 is proved.

Theorem 4.3. Assume that there exists $\bar{z} \in X$ such that $\bar{z} \leq x$ for all $x \in K$, that a space $\mathscr{A}$ is either $\mathcal{M}, \mathcal{M}_{v}$, or $\mathcal{M}_{c}$, and that $f_{a}=$ a for all $a \in \mathscr{A}$. Then there exists a set $\mathscr{F} \subset \mathscr{A}$ such that $\mathscr{A} \backslash \mathscr{F}$ is $\sigma$-porous in $\mathscr{A}$ with respect to $\left(d_{s}, d_{s}\right)$ and that for each $f \in \mathscr{F}$, the minimization problem for $f$ on $K$ is strongly well posed with respect to $\left(\mathscr{A}, d_{s}\right)$. If $K$ has the property $(C)$, then for each $f \in \mathscr{F}, \inf (f)$ is attained at a unique point.

Proof. We can prove Theorem 4.3 analogously to the proof of Theorem 4.2. The existence of a constant $\bar{d}$ is obtained in the following manner. Let $x \in K, u \in X$, $x \leq \bar{x}+u$, and $\|u\|<\epsilon$. Then

$$
\begin{gathered}
\|x\| \leq\|x-\bar{z}\|+\|\bar{z}\| \leq\|\bar{z}\|+\|\bar{x}+u-\bar{z}\| \leq 2 \mid \bar{z}\|+\| \bar{x} \|+\epsilon, \\
\|x\| \leq \bar{d}
\end{gathered}
$$

where $\bar{d}=2\|\bar{z}\|+\|\bar{x}\|+\epsilon$.

Denote by $\mathcal{M}^{+}$the set of all $f \in \mathcal{M}$ such that $f(x) \geq 0$ for all $x \in K$. Clearly, $M^{+}$is a closed subset of the metric space $\left(M, d_{s}\right)$. Define

$$
\begin{gathered}
M_{v}^{+}=M^{+} \cap M_{v}, \quad M_{c}^{+}=M^{+} \cap M_{c}, \quad M_{g}^{+}=M^{+} \cap M_{g}, \\
M_{g v}^{+}=M^{+} \cap M_{g v}, \quad M_{g c}^{+}=M^{+} \cap M_{g c} .
\end{gathered}
$$

For each $f, g \in \mathcal{M}^{+}$, set

$$
\begin{aligned}
& \tilde{d}_{w}(f, g)=\sup \{|\ln (f(z)+1)-\ln (g(z)+1)|: z \in K\}, \\
& d_{w}(f, g)=\tilde{d}_{w}(f, g)\left(1+\tilde{d}_{w}(f, g)\right)^{-1} .
\end{aligned}
$$

It is not difficult to see that the metrtic space $\left(\mathcal{M}^{+}, d_{w}\right)$ is complete and that $\mathcal{M}_{v}^{+}$, $M_{c}^{+}, M_{g}^{+}, M_{g v}^{+}$, and $M_{g c}^{+}$are closed subsets of $\left(M^{+}, d_{w}\right)$. Clearly, $d_{w}(f, g) \leq d_{s}(f, g)$ for all $f, g \in \mathcal{M}^{+}$. 
Theorem 4.4. Assume that one of the following cases holds:

(1) the space $\mathscr{A}$ is either $M_{g}^{+}, M_{g v}^{+}$, or $M_{g c}^{+}$;

(2) there is $\bar{z} \in X$ such that $\bar{z} \leq x$ for all $x \in K$ and that $\mathcal{A}$ is either $\mathcal{M}^{+}, \mathcal{M}_{v}^{+}$, or $M_{c}^{+}$.

Let $f_{a}=a$ for all $a \in \mathscr{A}$. Then there exists a set $\mathscr{F} \subset \mathscr{A}$ such that $\mathscr{A} \backslash \mathscr{F}$ is $\sigma$ porous in $\mathscr{A}$ with respect to $\left(d_{w}, d_{s}\right)$ and that for each $f \in \mathscr{F}$, the minimization problem for $f$ on $K$ is strongly well posed with respect to $\left(\mathscr{A}, d_{w}\right)$. If $K$ has the property $(C)$, then for each $f \in \mathscr{F}, \inf (f)$ is attained at a unique point.

Proof. By Theorems 3.1 and 3.2, we need to show that (H1), (H2), and (H3) hold and that the property (C) implies (H4). Clearly, (H1) holds. Analogously to the proofs of Theorems 4.2 and 4.3, we can show that (H2) is true. Therefore, in order to prove Theorem 4.4, it is sufficient to show that (H3) holds.

Let $m \geq 1$ be an integer. Choose $\alpha \in(0,1)$ such that

$$
\alpha<4^{-1}(2 e(m+1))^{-1} \text {. }
$$

Let $r \in(0,1], x \in K, f_{1}, f_{2} \in \mathscr{A}$,

$$
d_{w}\left(f_{1}, f_{2}\right) \leq \alpha r, \quad \min \left\{f_{1}(x), f_{2}(x)\right\} \leq m .
$$

We may assume without loss of generality that $f_{1}(x) \leq f_{2}(x)$. Then $\tilde{d}_{w}\left(f_{1}, f_{2}\right) \leq$ $2 d_{w}\left(f_{1}, f_{2}\right) \leq 2 \alpha r$

$$
\begin{gathered}
\ln \left(f_{2}(x)+1\right)-\ln \left(f_{1}(x)+1\right) \leq 2 \alpha r, \\
f_{2}(x)+1 \leq\left(f_{1}(x)+1\right) e^{2 \alpha r}, \\
\left|f_{2}(x)-f_{1}(x)\right| \leq\left(f_{1}(x)+1\right)\left(e^{2 \alpha r}-1\right) \\
\leq(m+1)\left(e^{2 \alpha r}-1\right)=2 \alpha r(m+1) e^{r_{1}},
\end{gathered}
$$

with $r_{1} \in[0,2 \alpha r]$. Then by (4.16),

$$
\left|f_{2}(x)-f_{1}(x)\right| \leq 2(m+1) \alpha r e^{2 \alpha r} \leq 2 \alpha e(m+1) r<r .
$$

Thus, (H3) holds and Theorem 4.4 is proved.

Fix a number $c_{0}>0$ and denote by $\mathcal{M}^{(\mathrm{co})}$ the set of all convex functions $f \in \mathcal{M}$ such that $f(x) \geq c_{0}\|x\|$ for all $x \in K$. Clearly, $\mathcal{M}^{(\mathrm{co})}$ is a closed subset of the metric space $\left(\mathcal{M}^{+}, d_{w}\right)$. Set $\mathcal{M}_{v}^{(\mathrm{co})}=\mathcal{M}^{(\mathrm{co})} \cap \mathcal{M}_{v}^{+}$and $\mathcal{M}_{c}^{(\mathrm{co})}=\mathcal{M}^{(\mathrm{co})} \cap \mathcal{M}_{c}^{+}$. Clearly, $\mathcal{M}_{v}^{(\mathrm{co})}$ and $\mathcal{M}_{c}^{(\mathrm{co})}$ are closed subsets of the metric space $\left(\mathcal{M}^{+}, d_{w}\right)$.

Theorem 4.5. Assume that a space $\mathcal{A}$ is either $\mathcal{M}^{\mathrm{co}}, \mathcal{M}_{v}^{(\mathrm{co})}$, or $\mathcal{M}_{c}^{(\mathrm{co})}$ and that $f_{a}=a$ for all $a \in \mathscr{A}$. Then there exists a set $\mathscr{F} \subset \mathscr{A}$ such that the complement $\mathscr{A} \backslash \mathscr{F}$ is $\sigma$ porous in $\mathscr{A}$ with respect to $\left(d_{w}, d_{w}\right)$ and that for each $f \in \mathscr{F}$, the minimization problem for $f$ on $K$ is strongly well posed with respect to $\left(\mathscr{A}, d_{w}\right)$. If the set $K$ has the property $(C)$, then for each $f \in \mathscr{F}, \inf (f)$ is obtained at a unique point. 


\section{Solutions of minimization problems}

Proof. By Theorems 3.1 and 3.2, we need to show that $(\mathrm{H} 1),(\mathrm{H} 2)$, and $(\mathrm{H} 3)$ hold and that the property (C) implies (H4). Clearly, (H1) holds. Analogously to the proof of Theorem 4.4, we can show that (H3) holds.

Now, we show that $(\mathrm{H} 2)$ holds and that the property $(\mathrm{C})$ implies $(\mathrm{H} 4)$.

Let $f \in \mathscr{A}, \epsilon \in(0,1)$, and $m \geq 1$ an integer. Choose a natural number $m_{0}$ and a number $r_{0}>0$ such that

$$
m_{0}>\left(m+c_{0}+2\right)\left(\min \left\{c_{0}, 1\right\}\right)^{-1}, \quad r_{0}<\left(m_{0}+2\right)^{-1} .
$$

Choose a positive number

$$
\delta<8^{-1} \in \min \left\{1, c_{0}\right\} .
$$

Assume that $f \in \mathscr{A}$,

$$
r \in\left(0, r_{0}\right], \quad \inf (f) \leq m .
$$

There exists $\bar{x} \in K$ such that

$$
f(\bar{x}) \leq \inf (f)+4^{-1} \delta r(m+1)^{-1} .
$$

If $K$ has the property (C), we assume that $\bar{x}$ is a minimal element of $K$. Define

$$
\bar{f}(x)=f(x)+4^{-1} r \min \left\{1, c_{0}\right\}(m+1)^{-1} \lambda(x-\bar{x}), \quad x \in K .
$$

It is easy to see that $\bar{f} \in \mathscr{A}$. It follows from (4.23) and (4.22) that

$$
c_{0}\|\bar{x}\| \leq f(\bar{x}) \leq \inf (f)+1 \leq m+1, \quad\|\bar{x}\| \leq(m+1) c_{0}^{-1} .
$$

By (4.14) and (4.24),

$$
\begin{aligned}
d_{w}(f, \bar{f}) \leq & \tilde{d}_{w}(f, \bar{f})=\sup \{|\ln (\bar{f}(x)+1)-\ln (f(x)+1)|: x \in K\} \\
& =\sup _{x \in K}\left\{\ln \left(1+(\bar{f}(x)-f(x))(f(x)+1)^{-1}\right)\right\} \\
\leq & \sup _{x \in K}\left\{\ln \left(1+\left(4^{-1} r \min \left\{1, c_{0}\right\} \lambda(x-\bar{x})\right)\left(\left(1+c_{0}\|x\|\right)(m+1)\right)^{-1}\right)\right\} \\
\leq & \sup _{x \in K}\left\{4^{-1} r \min \left\{1, c_{0}\right\} \lambda(x-\bar{x})\left(\left(1+c_{0}\|x\|\right)(m+1)\right)^{-1}\right\} \\
\leq & 4^{-1} r \sup _{x \in K}\left\{\min \left\{1, c_{0}\right\}\|x-\bar{x}\|\left(\left(1+c_{0}\|x\|\right)(m+1)\right)^{-1}\right\} \\
\leq & 4^{-1} r \sup _{x \in K}\left\{c_{0}\|x\|\left(1+c_{0}\|x\|\right)^{-1}\right. \\
& \left.\quad+\|\bar{x}\| \min \left\{1, c_{0}\right\}(m+1)^{-1}\right\} \leq 2^{-1} r .
\end{aligned}
$$

Thus

$$
d_{w}(f, \bar{f}) \leq 2^{-1} r
$$


Equation (4.24) and inequality (4.23) imply that

$$
\bar{f}(\bar{x})=f(\bar{x}) \leq \inf (f)+4^{-1} \delta r(m+1)^{-1} \leq \inf (\bar{f})+4^{-1} \delta r(m+1)^{-1} .
$$

Then by (4.22),

$$
\inf (\bar{f}) \leq \bar{f}(\bar{x})=f(\bar{x}) \leq \inf (f)+4^{-1} \delta r(m+1)^{-1} \leq m+1 .
$$

Let $x \in K$ and

$$
\bar{f}(x) \leq \inf (\bar{f})+4^{-1} \delta r(m+1)^{-1} .
$$

Inequality (4.30) and equation (4.29) imply that

$$
c_{0}\|x\| \leq m+2, \quad\|x\| \leq c_{0}^{-1}(m+2) .
$$

By (4.24), (4.30), (4.23), and (4.21),

$$
\begin{gathered}
\begin{aligned}
f(x) & +4^{-1} r(m+1)^{-1} \min \left\{1, c_{0}\right\} \lambda(x-\bar{x}) \\
& =\bar{f}(x) \leq \bar{f}(\bar{x})+4^{-1} \delta r(m+1)^{-1} \\
& =f(\bar{x})+4^{-1} \delta r(m+1)^{-1} \leq f(x)+2^{-1} \delta r(m+1)^{-1},
\end{aligned} \\
\min \left\{1, c_{0}\right\} \lambda(x-\bar{x}) \leq 2 \delta, \quad \lambda(x-\bar{x}) \leq 2 \delta\left(\min \left\{1, c_{0}\right\}\right)^{-1}<4^{-1} \epsilon .
\end{gathered}
$$

Therefore, there exists $u \in X$ such that $\|u\|<4^{-1} \epsilon$ and $x \leq \bar{x}+u$. Hence, (H2) is true and if $K$ has the property (C), then (H4) holds. This completes the proof of Theorem 4.5.

A function $f: K \rightarrow \mathbb{R}^{1} \cup\{\infty\}$ is called quasiconvex if the set $\{x \in K: f(x) \leq$ $\alpha\}$ is convex for any $\alpha \in \mathbb{R}^{1}$. Denote by $\mathcal{M}^{(\mathrm{qu})}$ the set of all quasiconvex functions $f \in M$. Clearly, $\mathcal{M}^{(\mathrm{qu})}$ is a closed subset of the metric space $\left(\mathcal{M}, d_{s}\right)$. Set

$$
\begin{gathered}
M_{v}^{(\mathrm{qu})}=\mathcal{M}^{(\mathrm{qu})} \cap \mathcal{M}_{v}, \quad \mathcal{M}_{c}^{(\mathrm{qu})}=\mathcal{M}^{(\mathrm{qu})} \cap \mathcal{M}_{c}, \quad M_{g}^{(\mathrm{qu})}=\mathcal{M}^{(\mathrm{qu})} \cap M_{g}, \\
M_{g v}^{(\mathrm{qu})}=\mathcal{M}^{(\mathrm{qu})} \cap \mathcal{M}_{g v}, \quad M_{g c}^{(\mathrm{qu})}=\mathcal{M}^{(\mathrm{qu})} \cap \mathcal{M}_{g c} .
\end{gathered}
$$

Theorem 4.6. Assume that one of the following cases holds:

(1) a space $\mathcal{A}_{\text {is }}$ either $M_{g}^{(\mathrm{qu})}, M_{g v}^{(\mathrm{qu})}$, or $M_{g c}^{(\mathrm{qu})}$;

(2) there is $\bar{z} \in X$ such that $\bar{z} \leq x$ for all $x \in K$ and a space $\mathscr{A}$ is either $\mathcal{M}^{\text {(qu), }}$, $M_{v}^{(\mathrm{qu})}$, or $M_{c}^{(\mathrm{qu})}$.

Let $f_{a}=a$ for all $a \in \mathscr{A}$. Then there exists a set $\mathscr{F} \subset \mathcal{A}$ such that the complement $\mathscr{A} \backslash \mathscr{F}$ is $\sigma$-porous in $\mathscr{A}$ with respect to $\left(d_{s}, d_{s}\right)$ and that for each $f \in \mathscr{F}$, the minimization problem for $f$ on $K$ is strongly well posed with respect to $\left(\mathscr{A}, d_{s}\right)$. If $K$ has the property $(C)$, then for each $f \in \mathscr{F}, \inf (f)$ is attained at a unique point. 
Proof. By Theorems 3.1 and 3.2, we need to show that $(\mathrm{H} 1),(\mathrm{H} 2)$, and $(\mathrm{H} 3)$ hold and that the property (C) implies (H4). Clearly, (H1) holds. Analogously to the proof of Theorem 4.2, we can show that $(\mathrm{H} 3)$ is true (see (4.7)). Now, we show that $(\mathrm{H} 2)$ holds and that the property $(\mathrm{C})$ implies $(\mathrm{H} 4)$.

Let $f \in \mathscr{A}, \epsilon \in(0,1]$, and $r \in(0,1]$. Choose $\bar{x} \in K$ such that

$$
f(\bar{x}) \leq \inf (f)+\frac{\epsilon r}{8} .
$$

If $K$ has the property (C), then we assume that $\bar{x}$ is a minimial element of $K$. Define

$$
\bar{f}(x)=\max \left\{f(x), f(\bar{x})+2^{-1} r \min \{\lambda(x-\bar{x}), 1\}\right\}, \quad x \in K .
$$

Clearly, $\bar{f} \in \mathscr{A}$,

$$
\begin{gathered}
d_{s}(f, \bar{f}) \leq \tilde{d}_{s}(f, \bar{f}) \leq r \\
\bar{f}(\bar{x})=f(\bar{x}) \leq \inf (f)+\frac{\epsilon r}{8} \leq \inf (\bar{f})+\frac{\epsilon r}{8}, \\
\inf (\bar{f}) \leq \bar{f}(\bar{x})=f(\bar{x}) \leq \inf (f)+1
\end{gathered}
$$

Let $x \in K$ and

$$
\bar{f}(x) \leq \inf (\bar{f})+\frac{\epsilon r}{8} .
$$

Then by (4.35) and (4.37),

$$
\begin{gathered}
2^{-1} r \min \{\lambda(x-\bar{x}), 1\}+f(\bar{x}) \leq \bar{f}(x) \leq \bar{f}(\bar{x})+\frac{\epsilon r}{8}=f(\bar{x})+\frac{\epsilon r}{8}, \\
\min \{\lambda(x-\bar{x}), 1\} \leq \frac{\epsilon}{4}, \quad \lambda(x-\bar{x}) \leq \frac{\epsilon}{4},
\end{gathered}
$$

and there exists $u \in X$ such that

$$
\|u\|<\frac{\epsilon}{2}, \quad x \leq \bar{x}+u .
$$

In the first case, we choose $\bar{d}>0$ such that $\bar{f}(y)>\inf (\bar{f})+1$ for all $y \in K$ satisfying $\|y\|>\bar{d}$ and obtain that $\|x\| \leq \bar{d}$. In the second case,

$$
\begin{gathered}
\bar{z} \leq x \leq \bar{x}+u \\
\|x\| \leq\|\bar{z}\|+\|x-\bar{z}\| \leq\|\bar{z}\|+\|\bar{x}+u-\bar{z}\| \\
\leq 2\|\bar{z}\|+\|\bar{x}\|+\|u\| \leq 2\|\bar{z} \mid+\| \bar{x} \|+1,
\end{gathered}
$$

and $\|x\| \leq \bar{d}:=2\|\bar{z}\|+\|\bar{x}\|+1$. Therefore, in both cases, (H2) is true and if the set $K$ has the property (C), then (H4) holds. Theorem 4.6 is proved. 
Theorem 4.7. Let $\mathscr{A}$ be defined as in Theorem 4.6 and let $\mathscr{A}^{+}$be the set of all $f \in \mathscr{A}$ such that $f(x) \geq 0$ for all $x \in K$. Assume that $f_{a}=a$ for all $a \in \mathscr{A}^{+}$. Then the metric spaces $\left(\mathscr{A}^{+}, d_{s}\right)$ and $\left(\mathscr{A}^{+}, d_{w}\right)$ are complete and there exists a set $\mathscr{F} \subset \mathscr{A}^{+}$ such that the complement $\mathscr{A}^{+} \backslash \mathscr{F}$ is $\sigma$-porous in $\mathscr{A}^{+}$with respect to $\left(d_{w}, d_{s}\right)$ and that for each $f \in \mathscr{F}$, the minimization problem for $f$ on $K$ is strongly well posed with respect to $\left(\mathscr{A}^{+}, d_{w}\right)$. If the set $K$ has the property $(C)$, then for each $f \in \mathscr{F}$, $\inf (f)$ is attained at a unique point.

Proof. By Theorems 3.1 and 3.2, we need to show that $(\mathrm{H} 1),(\mathrm{H} 2)$, and $(\mathrm{H} 3)$ hold and that the property (C) implies (H4). Clearly, (H1) holds. Analogously to the proof of Theorem 4.6, we can show that $(\mathrm{H} 2)$ is true and that the property (C) implies (H4). We can prove (H3) as in the proof of Theorem 4.4.

\section{References}

[1] G. Beer and R. Lucchetti, Convex optimization and the epi-distance topology, Trans. Amer. Math. Soc. 327 (1991), no. 2, 795-813.

[2] F. S. De Blasi and J. Myjak, On a generalized best approximation problem, J. Approx. Theory 94 (1998), no. 1, 54-72.

[3] R. Deville, G. Godefroy, and V. Zizler, Smoothness and Renormings in Banach Spaces, Pitman Monographs and Surveys in Pure and Applied Mathematics, vol. 64, Longman Scientific \& Technical, Harlow, 1993.

[4] R. Deville and J. P. Revalski, Porosity of ill-posed problems, Proc. Amer. Math. Soc. 128 (2000), no. 4, 1117-1124.

[5] A. L. Dontchev and T. Zolezzi, Well-Posed Optimization Problems, Lecture Notes in Mathematics, vol. 1543, Springer-Verlag, Berlin, 1993.

[6] A. D. Ioffe and A. J. Zaslavski, Variational principles and well-posedness in optimization and calculus of variations, SIAM J. Control Optim. 38 (2000), no. 2, 566-581.

[7] M. A. Krasnosel'skiǐ, Positive Solutions of Operator Equations, P. Noordhoff, Groningen, 1964.

[8] S. Reich and A. J. Zaslavski, On the minimization of convex functionals, Calculus of Variations and Differential Equations (Haifa, 1998), Chapman \& Hall/CRC Res. Notes Math., vol. 410, Chapman \& Hall, Florida, 2000, pp. 200-209.

[9] J. P. Revalski, Generic properties concerning well-posed optimization problems, C. R. Acad. Bulgare Sci. 38 (1985), no. 11, 1431-1434.

[10] A. M. Rubinov and A. J. Zaslavski, Existence and uniqueness of a solution for a minimization problem with a generic increasing function, J. Austral. Math. Soc. Ser. A 67 (1999), no. 1, 85-103.

[11] H. H. Schaefer, Banach Lattices and Positive Operators, Die Grundlehren der mathematischen Wissenschaften, vol. 215, Springer-Verlag, New York, 1974.

[12] A. J. Zaslavski, Generic existence of solutions of minimization problems with an increasing cost function, to appear in Nonlinear Funct. Anal. Appl.

[13] , Generic well-posedness of optimal control problems without convexity assumptions, SIAM J. Control Optim. 39 (2000), no. 1, 250-280.

[14]_, Existence of solutions of optimal control problems for a generic integrand without convexity assumptions, Nonlinear Anal., Ser. A: Theory Methods 43 (2001), no. 3, 339-361. 
670 Solutions of minimization problems

[15] Well-posedness and porosity in optimal control without convexity assumptions, Calc. Var. Partial Differential Equations 13 (2001), no. 3, 265-293.

Alexander J. Zaslavski: Department of Mathematics, The Technion-Israel Institute of Technology, 32000 Haifa, Israel

E-mail address: ajzasl@tx.technion.ac.il 


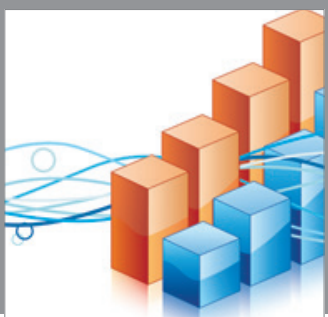

Advances in

Operations Research

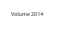

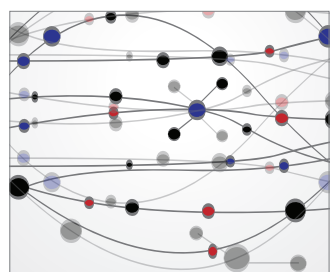

\section{The Scientific} World Journal
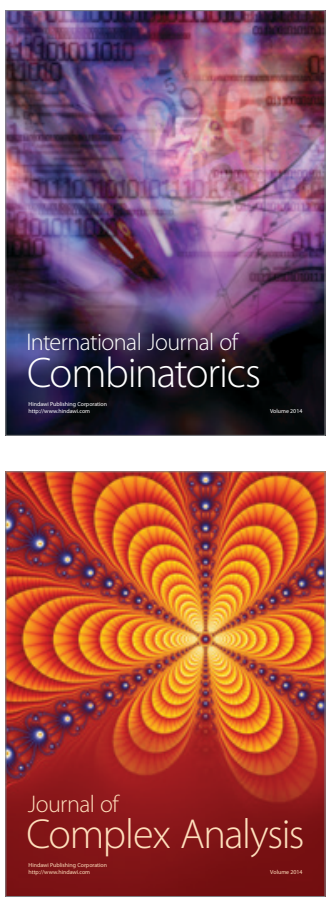

International Journal of

Mathematics and

Mathematical

Sciences
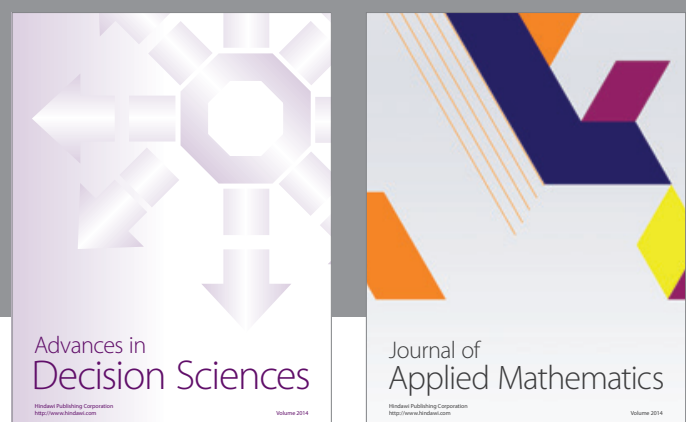

Journal of

Applied Mathematics
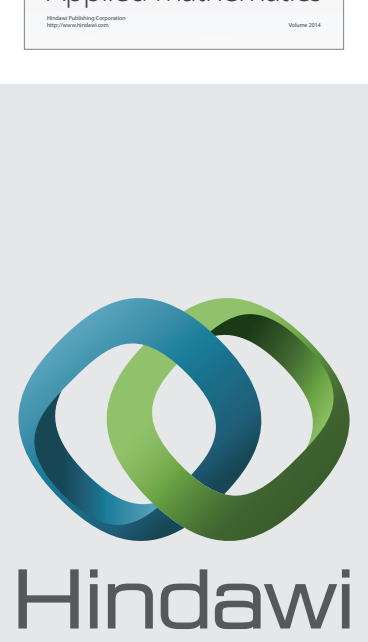

Submit your manuscripts at http://www.hindawi.com
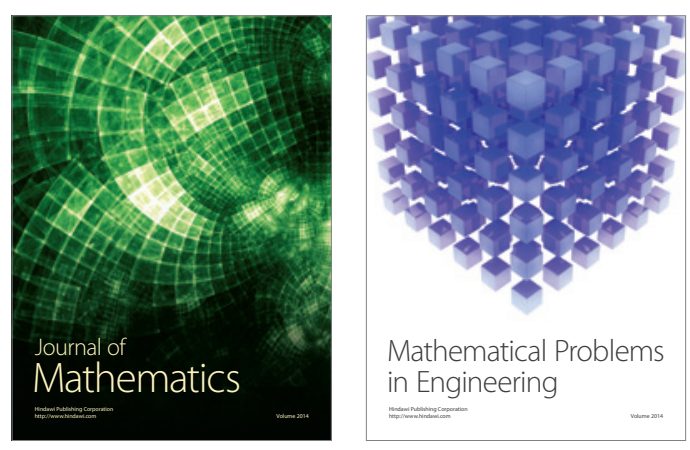

Mathematical Problems in Engineering
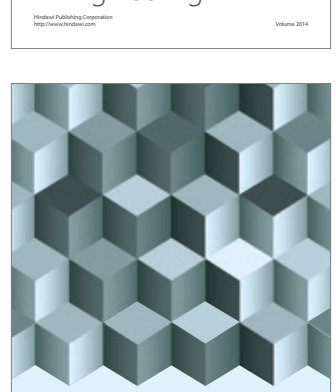

Journal of

Function Spaces
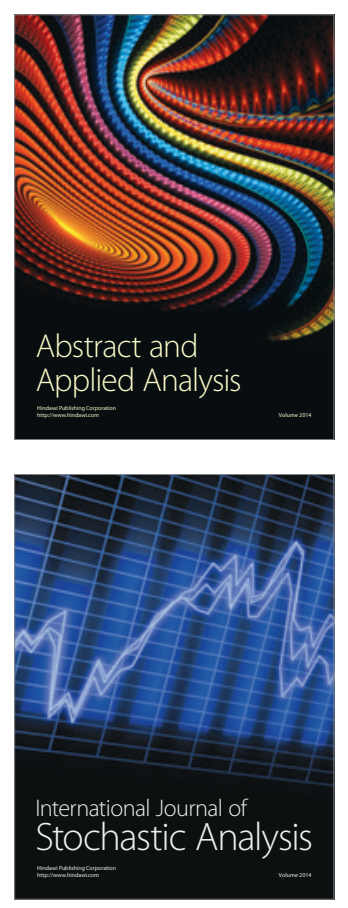

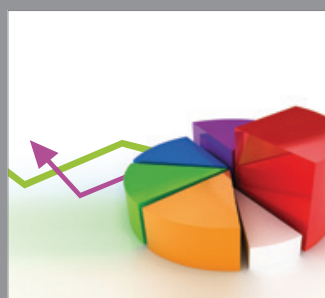

ournal of

Probability and Statistics

Promensencen
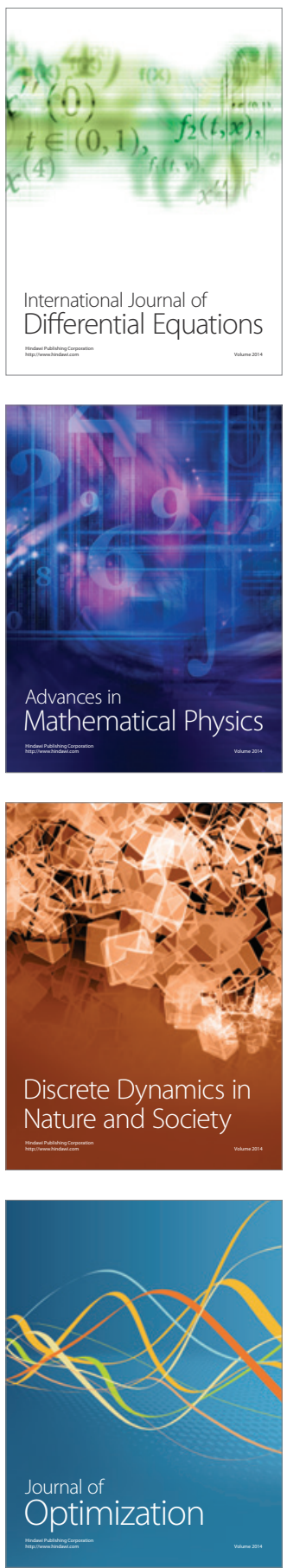